\title{
Présentation de la série du GECO de métastases osseuses des membres : 90 cas
}

\section{The series of the GECO group: 90 cases}

P. Kehr, G. Copin, P. Grumillier, J.H. Jaeger, M. Jandeaux, M. Perrin et J.P. Steib

Hôpital Stéphanie, F-67026 Strasbourg Cedex

\begin{abstract}
This series of surgical patients shows that $50 \%$ of bony metastases are complicated by a fracture. Of these, 50\% occurred without any preceding symptoms or signs.
\end{abstract}

Key words : Metastases - Bone

Résumé. Dans cette série de cas, vus par des chirurgiens, une métastase sur deux est traitée au stade de fracture. Une fracture sur deux est survenue en l'absence de toute symptomatologie évocatrice.

Mots-clés : Métastases osseuses

Voici les résultats de l'enquête effectuée dans les différents services de notre groupe à l'occasion de ce travail.

Conditions de la découverte (90)

$\begin{array}{lrr}\text { bilan initial : } & 4 \% & (4) \\ \text { surveillance } & 12 \% & (11) \\ \text { douleur } & \mathbf{4 2} \% & \mathbf{( 3 8 )} \\ \text { fracture } & \mathbf{4 1 \%} & \mathbf{( 3 7 )}\end{array}$

Les douleurs n'imposent la consultation avant la fracture qu'une fois sur deux.

Localisations

$\begin{array}{lrr}\text { humérus } & 18 \% & (16) \\ \text { tête } & & (2) \\ 1 / 3 \text { moyen } & & (14)\end{array}$

Code Méary : 0499.1

$\begin{array}{lrr}\text { radius } & \mathbf{1} \% & (\mathbf{1}) \\ \text { bassin } & \mathbf{1 \%} & (\mathbf{1}) \\ \text { fémur } & 73 \% & (\mathbf{6 6}) \\ \text { col } & & (11) \\ \text { massif trochantérien } & & (2) \\ 1 / 3 \text { moyen } & & (39) \\ \text { supra-condyliennes } & & (2) \\ \text { tibia } & 7 \% & (6)\end{array}$

Les diaphyses humérales et surtout fémorales sont les segments osseux les plus souvent atteints.

Diagnostic de certitude

$\begin{array}{lrr}\text { scintigraphie } & 2 \% & (2) \\ \text { métastase attendue } & 27 \% & (24) \\ \text { marqueurs } & 1 \% & (1) \\ \text { histologie } & 17 \% & (15) \\ \text { radiographie } & \mathbf{5 0} \% & (45) \\ \text { inconnu } & 3 \% & (3)\end{array}$

Il ne faut pas engager de traitement sans preuve. L'histologie est utile pour les métastases précessives et les cas douteux : au total $17 \%$.

\section{Douleur}

$\begin{array}{lrr}\text { nulle } & 2 \% & (2) \\ \text { modérée } & 11 \% & (10) \\ \text { moyenne } & 12 \% & (11) \\ \text { importante } & \mathbf{5 6 \%} & \mathbf{5 0 )} \\ \text { réfractaire } & 9 \% & (8) \\ \text { inconnue } & 10 \% & (9)\end{array}$

\section{Risque fracturaire}

Sans objet : car déjà fracturée $\quad 41 \%$ Intéressant: $\quad 59 \%$

$\begin{array}{lll}\text { nul } & 1 \% & (1) \\ \text { modéré } & 8 \% & (4) \\ \text { moyen } & 8 \% & (4)\end{array}$

$\begin{array}{lrr}\text { important } & 28 \% & (15) \\ \text { imminent } & \mathbf{4 5 \%} & \mathbf{( 2 4 )} \\ \text { inconnu } & 10 \% & (5)\end{array}$

Les indications trop précoces semblent rares. Il existe une bonne corrélation entre la symptomatologie, qui oblige à consulter, et l'imminence du risque fracturaire.

\section{Invalidité}

$\begin{array}{lcr}\text { inconnue } & 6 \% & (5) \\ \text { nulle } & 0 & \\ \text { mineure } & 9 \% & (8) \\ \text { gène notable } & \mathbf{2 0 \%} & \mathbf{( 1 8 )} \\ \text { gène importante } & \mathbf{2 7} \% & \mathbf{( 2 4 )} \\ \text { grabataire } & \mathbf{3 7} \% & \mathbf{( 3 3 )}\end{array}$

Là encore il existe une bonne corrélation entre les signes d'appel et le risque fracturaire.

\section{Etat antérieur sur 37 fractures}

$\begin{array}{lrr}\text { normal } & \mathbf{4 3} \% & (\mathbf{1 6}) \\ \text { quelques douleurs } & 27 \% & (10) \\ \text { marche difficile } & 19 \% & (7) \\ \text { grabataire } & 5 \% & (2) \\ \text { impossible à mobiliser } & 5 \% & (2)\end{array}$

Une fois sur deux la fracture survient sur un sujet indemne de toute symptomatologie. L'élément traumatique n'est pas négligeable mais on constate que certaines métastases osseuses très évoluées sont asymptomatiques. Les surveillances systématiques sont donc utiles, surtout lorsque la diffusion osseuse est connue et qu'elle évolue sans douleur. 